\title{
RIEMANN-ROCH THEOREM FOR OPERATIONS IN COHOMOLOGY OF ALGEBRAIC VARIETIES
}

\author{
A. L. SMIRNOV
}

\begin{abstract}
The Riemann-Roch theorem for multiplicative operations in oriented cohomology theories for algebraic varieties is proved and an explicit formula for the corresponding Todd classes is given. The formula obtained can also be applied in the topological situation, and the theorem can be regarded as a change-of-variables formula for the integration of cohomology classes.
\end{abstract}

The classical Riemann-Roch theorem [1], stated and proved by Hirzebruch, calculates the Euler characteristic of a vector bundle on a smooth projective algebraic variety $X / \mathbb{C}$ in terms of its rank and Chern classes. This theorem states that $\chi(E)$ coincides with the $2 n$th component of the characteristic class $\operatorname{ch}(E) \operatorname{td}(X)$ under the identification $H^{2 n}(X(\mathbb{C}), \mathbb{Q})=\mathbb{Q}$, where $n=\operatorname{dim} X, \operatorname{ch}(E)$ is the Chern character of $E$, and $\operatorname{td}(X)$ is the Todd class of the tangent bundle $T_{X}$. By the splitting principle, the calculation of the Todd classes reduces to the case of a line bundle $L$, where $\operatorname{td}(L)$ is obtained by substituting $z=c_{1}(L)$ in the series

$$
\operatorname{td}(z)=\frac{z}{1-e^{-z}}
$$

Grothendieck's generalization of the Riemann-Roch theorem [2] deals with the ring $K_{0}$ of virtual bundles and calculates the rank and rational Chern classes of a certain virtual bundle, namely, of the direct image of $E$ under a proper mapping $p: X \rightarrow Y$ (the Hirzebruch theorem is obtained if $Y=\mathrm{pt}$, and $\chi(E)$ is regarded as the rank of the direct image in $K_{0}$ ). After the functor $K_{0}$ was carried over to topology and was extended to a cohomology theory, the corresponding version of the Riemann-Roch theorem arose [3].

A more general topological Riemann-Roch theorem appeared in the paper [4] by E. Dyer, where he mentioned the folklore origin of the theorem and the fact that the theorem was known to Adams, Atiyah, and Hirzebruch. In that theorem, the Chern character is replaced by an arbitrary multiplicative operation between arbitrary cohomology theories (the varieties $X$ and $Y$ were assumed to be oriented in each of the two cohomology theories).

When motivic versions of topological theories appeared $[5,6,7]$ and transfers for oriented theories were constructed [8] (see [9, 10] for a complete account), a natural problem arose: to generalize the Riemann-Roch theorem to the motivic situation. Such a theorem was stated, and two proofs of it were outlined in [8].

In the present paper, we present the first of these proofs. This proof involves a direct calculation based on the invariance of the residue. The corresponding Todd classes are defined by a simple formula (see Definition 2.2.1) generalizing (1). We show that the same formula can be used in the topological setting. Furthermore, the Riemann-Roch

2000 Mathematics Subject Classification. Primary 14F25, 14F42, 14F43, 14 F99.

Key words and phrases. Algebraic variety, oriented cohomology theory, transfer, characteristic class, Todd class, Riemann-Roch theorem.

Supported by RFBR (grant 06-01-00741) 
theorem is regarded as a change-of-variables formula in an integral. For example, for a smooth projective variety $X$, the theorem can be given in the form

$$
\phi \int_{X} \alpha \theta=\int_{X} \phi(\alpha) j_{\phi} \eta
$$

where $\phi: A \rightarrow B$ is a multiplicative operation between oriented theories, $\theta$ and $\eta$ are the volume forms on $X$ defined by the orientations of $A$ and $B, j_{\phi}$ is the value of the Jacobian of $\phi$ on the normal bundle of $X, \alpha$ is a cohomology class, and the integral is the direct image operator.

Like the change-of-variables formula in integrals, the Riemann-Roch theorem itself does not calculate anything. Both formulas are effective since they reduce complicated integrals to simpler ones.

The second of the above-mentioned proofs of the Riemann-Roch theorem, based on the uniqueness of the transfers compatible with an orientation, was presented in [11]. The approach developed in that paper can be applied to partially defined theories and operations.

Now, we describe the content of the present paper in more detail. In Subsection 1.1, we give necessary information concerning cohomology theories. In Subsection 1.2, we introduce the notions of an operation and a quasistable operation. In Subsection 1.3, we consider examples of operations.

In Subsection 2.1, we present some facts about transfers and oriented theories. In Subsection 2.2, we introduce the Todd series $\operatorname{td}_{\phi}$, the Jacobian $j_{\phi}$, and the corresponding characteristic classes. In Subsection 2.3, we present some facts concerning the interaction between an operation and differential forms or their residues; in particular, we calculate the action of an operation on the invariant form of the corresponding formal group. In Subsection 2.4, we prove two versions of the Riemann-Roch theorem. Theorem 2.4.1 can be applied to an arbitrary operation, but only for embeddings. The main result of the present paper is Theorem 2.4.3. This version applies to an arbitrary mapping, but only to a quasistable operation. In Subsection 2.5, we consider several examples.

In Subsection 3.1, we construct a transfer that does not depend on the choice of an orientation (in topology, the existence of such a transfer follows from the Milnor-Spanier duality). In Subsection 3.2, we compare the Riemann-Roch theorem and the change-ofvariables formula in integrals and show that the series $j_{\phi}$ can be regarded as the Jacobian of the change of parameters on $\mathbf{P}^{\infty}$. In Subsection 3.3, we show that the formulas for the Todd classes in Subsection 2.2.5 are applicable in the topological situation.

The author wants to thank N. Yu. Netsvetaev who pointed at the paper [4] as a source of the topological Riemann-Roch theorem.

\section{$\S 1$. Cohomology theORIES And Operations}

We use the following terminology. A variety is an arbitrary, possibly nonsmooth, quasiprojective variety over a fixed field $k$; pt $=\operatorname{Spec} k$; an embedding of varieties is a locally closed embedding; a smooth variety is an equidimensional smooth variety; a vector bundle is a vector bundle of constant rank; a space is a smooth open pair.

1.1. Cohomology theory. In this paper, we deal with cohomology theories that are commutative, spherically stable, orientable ring theories [10]. Below, we present some facts concerning these theories.

1.1.1. The target category. A ring theory takes values in a category $\mathcal{A}$ equipped with a system of exact triangles and a compatible structure of an additive symmetric monoidal category with unit $R$. The fact that a category is equipped with a system of exact triangles means that $\mathcal{A}$ is an additive category (the morphisms of $\mathcal{A}$ are called 
operators) equipped with a shift, i.e., with an invertible additive endofunctor $M \mapsto M[1]$ $(M[n]$ is the result of the shift applied $n$ times) and with a collection of triangles called exact (a triangle is a diagram of the form $X \stackrel{u}{\rightarrow} Y \stackrel{v}{\rightarrow} Z \stackrel{w}{\rightarrow} X[1]$, where $v u=0, w v=0$, and $u[1] \circ w=0)$. Such a collection must satisfy certain axioms [10], which are not needed here because, in our basic examples, the systems of exact triangles are given explicitly.

The compatibility of a system of exact triangles with the structure of an additive symmetric monoidal category means that the shift in $\mathcal{A}$ is performed by left multiplication by the invertible object $R[1]$, and the functor $M \mapsto \operatorname{Hom}(R, M)$ takes exact triangles to exact sequences.

In our examples, $\mathcal{A}$ is the category of bigraded modules over a commutative bigraded ring $R$ (a commutative monoid in the tensor category of bigraded Abelian groups with the permutation $x \otimes y \mapsto(-1)^{d} y \otimes x$, where $d=\operatorname{deg}_{1} x \operatorname{deg}_{1} y$, on the homogeneous elements). This category is equipped with the shift $M[1]^{i, j}=M^{i+1, j}$, and a triangle is exact if the sequences induced by this triangle in each degree are exact.

To study the operations between cohomology theories, we use the following convenient terminology.

1.1.2. Monoidal functors. A functor is weakly monoidal if it satisfies all conditions $[13,4.1 .2]$ of a monoidal functor except the invertibility of $F(X) \otimes F(Y) \rightarrow F(X \otimes Y)$. In other words, a weakly monoidal functor is a triple $(F, m, \alpha)$, where $F: \mathcal{C} \rightarrow \mathcal{D}$ is a functor of monoidal categories, $m: F(X) \otimes F(Y) \rightarrow F(X \otimes Y)$ is a morphism of functors, and $\alpha: F\left(1_{\mathcal{C}}\right) \rightarrow 1_{\mathcal{D}}$ is an isomorphism. Here, two morphisms from $(F X \otimes F Y) \otimes F Z$ to $F\left(X \otimes(Y \otimes Z)\right.$ ), two morphisms from $F\left(1_{\mathcal{C}}\right) \otimes F X$ to $F\left(1_{\mathcal{C}} \otimes X\right)$, and two morphisms from $F X \otimes F\left(1_{\mathcal{C}}\right)$ to $F\left(X \otimes 1_{\mathcal{C}}\right)$ must coincide. For symmetric categories $\mathcal{C}$ and $\mathcal{D}$, a weakly monoidal functor is said to be symmetric if $F\left(t_{\mathcal{C}}\right) m=m t_{\mathcal{D}}$, where $t_{\mathcal{C}}$ and $t_{\mathcal{D}}$ are permutations of the symmetric structures. Neglecting some subtleties, we assume that $F\left(1_{\mathcal{C}}\right)=1_{\mathcal{D}}$, which allows us to avoid mentioning $\alpha$.

A morphism of weakly monoidal (symmetric) functors is defined in the same way as a morphism of monoidal (symmetric) functors; see [13, 4.1.5].

1.1.3. Commutative ring theories. A cohomology theory with values in $\mathcal{A}$ (see $[10,1.2])$ consists of a contravariant functor $A(X, U)$ from the category of smooth open pairs to the category $\mathcal{A}$ and a morphism of functors $\partial: A(U) \rightarrow A(X, U)[1]$ satisfying the following conditions: (a) localization: a triangle $A(X, U) \rightarrow A(X) \rightarrow A(U) \rightarrow$ $A(X, U)[1]$, where the operators are given by the embeddings $(X, \varnothing) \rightarrow(X, U)$ and $U \rightarrow X$ and the differential, is exact; (b) excision: the operator $A(X, U) \rightarrow A(\tilde{X}, \tilde{U})$ induced by the morphism $e:(\tilde{X}, \tilde{U}) \rightarrow(X, U)$ is an isomorphism if $e: \tilde{X} \rightarrow X$ is étale at all points of $\tilde{Z}=\tilde{X}-\tilde{U}$ and induces an isomorphism between $\tilde{Z}$ and $Z=X-U$; (c) homotopy invariance: the operator $A(X) \rightarrow A\left(X \times \mathbf{A}^{1}\right)$ induced by the projection $X \times \mathbf{A}^{1} \rightarrow X$ is an isomorphism.

In the sequel, we assume that the cohomology theory is a commutative ring theory (see $[10,1.4])$. This means that $A$ is equipped with a structure of a symmetric weakly monoidal functor $m: A(P) \otimes A(Q) \rightarrow A(P \times Q)$ such that $\partial \otimes 1_{Y}=\partial_{(X, U) \times Y}$ for each pair $(X, U)$ and a smooth variety $Y$. The tensor product in $\mathcal{A}$ is the tensor product over $R=A(\mathrm{pt})$.

Many results are stated naturally in terms of spaces of type $\mathbf{P}^{\infty}$, which, strictly speaking, are not varieties. For this, we use the extension of the cohomology theory by the ind- and pro-objects; see [10, 4.3]. The same extension is used for operations.

1.1.4. Spherically stable theories. In what follows, we consider only spherically stable theories $[10,1.5]$. This means that the product $C \otimes A(Y) \rightarrow A\left(\Sigma_{a} \times Y\right)$ is an isomorphism for all $Y$, the functor $M \mapsto C \otimes M$ takes the exact triangles to exact 
triangles, and $C=A\left(\Sigma_{a}\right)$, where $\Sigma_{a}=\left(\mathbf{A}^{1}, \mathbf{A}^{1}-0\right)$, is invertible. In particular, we have the twists $M[p](q)=R[1]^{\otimes p} \otimes R(1)^{\otimes q} \otimes M$, where, by definition, $R(-1)=R[2] \otimes C$, so that $C=R[-2](-1)$. For the category of bigraded modules, we assume that $M(1)^{i, j}=$ $M^{i, j+1}$.

1.2. Operations. Let $A$ and $B$ be cohomology theories with values in categories $\mathcal{A}$ and $\mathcal{B}$, respectively (see Subsection 1.1). Since the theories take values in different categories, we need a functor from one category to the other to be able to define operations between the theories. To study unstable operations (Subsection 1.2.2), it is convenient to view $R(1)$ as a part of the structure of a target category.

Let $R$ be an identity in the category $\mathcal{A}, S$ an identity in the category $\mathcal{B}, C_{A}=A\left(\Sigma_{a}\right)$, and $C_{B}=B\left(\Sigma_{a}\right)$. The pullback operators $f^{*}$ related to the theories $A$ and $B$ will be denoted by $f^{A}$ and $f^{B}$, respectively.

1.2.1. Comparison of target categories. A comparison functor for target categories is a symmetric monoidal additive functor $\tilde{F}=(F, m): \mathcal{A} \rightarrow \mathcal{B}$ (see Subsection 1.1.2) equipped with isomorphisms $\alpha^{1,0}: F(R[1]) \rightarrow S[1]$ and $\alpha^{0,1}: F(R(1)) \rightarrow S(1)$. For brevity, we denote such a functor by $F$.

The comparison functor gives rise to an isomorphism $\alpha^{i, j}: F(M[i](j)) \rightarrow M[i](j)$ defined by the composition $F(N[p](q)) \rightarrow F(N) F(R[1])^{\otimes p} F(R(1))^{\otimes q} \rightarrow F(N)[p](q)$, where the first arrow is inverse to the operator $F(N) \otimes F(R[1])^{\otimes p} \otimes F(R(1))^{\otimes q} \rightarrow F(N[p](q))$ that is induced by $m$ and invertible because $F$ is monoidal. The second arrow is the operator $1 \otimes \alpha_{1,0}^{\otimes i} \otimes \alpha_{0,1}^{\otimes j}$.

Moreover, for each operator of degree $[p](q)$ from $M$ to $N$, i.e., for an operator $g$ : $M \rightarrow N[p](q)$ in the category $\mathcal{A}$, we have the following operator of degree $[p](q)$ in the category $\mathcal{B}$ :

$$
\tilde{F}(g): F(M) \stackrel{F(g)}{\longrightarrow} F(N[p](q)) \stackrel{\alpha^{p, q}}{\longrightarrow} F(N)[p](q) .
$$

This notation allows us to distinguish between the operator mentioned above and $F g$ and emphasizes its dependence on the $\alpha$-data of the comparison functor.

The following definition introduces the notion of an operation. For brevity, we use this name for an object that should be called a multiplicative operation.

1.2.2. Definition. An operation $A \rightarrow B$ is a comparison functor for target categories $\tilde{F}: \mathcal{A} \rightarrow \mathcal{B}$ (Subsection 1.2.1) and a morphism of functors $\phi: F \circ A \rightarrow B$ for which the following diagrams are commutative:

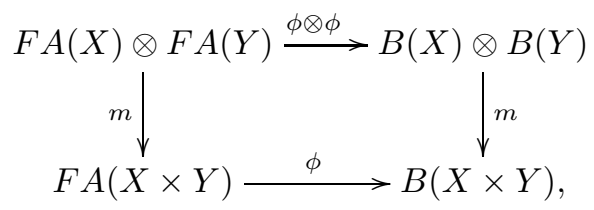

where $X$ and $Y$ are arbitrary spaces. For brevity, we denote an operation by $\phi$ and the operator $\phi(X): F A(X) \rightarrow B(X)$ by $\phi$ or by $\phi_{X}$.

An operation $\phi$ is said to be quasistable if $\phi_{X}$ is an isomorphism, and stable if $\phi_{X}=$ $\alpha^{2,1}$ (see Subsection 1.2.1) for $X=\Sigma_{a}$ (see Subsection 1.1.4).

An operation $\phi$ is quasistable if and only if $\phi_{\mathbf{P}^{1}}$ is an isomorphism, because we have canonically $A\left(\mathbf{P}^{1}\right)=R+C_{A}\left[10\right.$, Subsection 1.5.1], and $\phi_{\mathrm{pt}}$ is an isomorphism.

1.2.3. The action of an operation on $A^{p, q}(X)$. An operation $\phi$ induces a homomorphism $\phi_{X}: A^{p, q}(X) \rightarrow B^{p, q}(X)$ of Abelian groups defined by the composition $\phi \circ$ $\left(\alpha^{-p,-q}\right)^{*} \circ F$, where

$$
A^{p, q}(X)=\operatorname{Hom}(R, A(X)[p](q))=\operatorname{Hom}(R[-p](-q), A(X)) .
$$


We have $\phi_{Y} \circ f^{A}=f^{B} \circ \phi_{X}$ for a mapping $f: X \rightarrow Y$ of spaces, $\phi_{\mathrm{pt}}(1)=1$, and $\phi(x \cdot y)=\phi(x) \cdot \phi(y)$.

The study of quasistable operations reduces to the study of stable ones because a quasistable operation $\phi=\left(F, m, \alpha^{p, q}, \phi\right)$ induces a stable operation $\tilde{\phi}=\left(F, m, \tilde{\alpha}^{p, q}, \tilde{\phi}\right)$, where $\tilde{\phi}=\phi, \tilde{\alpha}^{p, q}=\lambda^{-p} \mu^{-q} \alpha^{p, q}$, and $\lambda, \mu \in S^{0,0}$ are such that $\phi_{\Sigma_{a}}=\lambda^{2} \mu \alpha^{2,1}$.

1.2.4. Elementary description of operations. Let $\mathcal{A}$ and $\mathcal{B}$ be categories of twosided $R$-modules and $S$-modules over commutative bigraded rings (see Subsection 1.1.1). We assume that, for each space $X$, we have a homomorphism of bigraded Abelian groups $\phi_{X}: A(X) \rightarrow B(X)$ such that the operators $\phi_{X}$ commute with pullbacks and are multiplicative in the sense that $\phi(x y)=\phi(x) \phi(y)$ and $\phi(1)=1$.

These data determine an operation as follows: $F(M)=S \otimes_{R} M$, where the structure of an $R$-algebra on $S$ is given by the homomorphism $\phi_{\mathrm{pt}}$, the operator $m: F(M) \otimes F(N) \rightarrow$ $F(M \otimes N)$ is given by the formula $m[(s \otimes u) \otimes(t \otimes v)]=(-1)^{d} s t \otimes(u \otimes v)$, where $t$ and $u$ are homogeneous elements of $S$ and $M$, and $d=\operatorname{deg}_{1} t \operatorname{deg}_{1} u+\operatorname{deg}_{2} t \operatorname{deg}_{2} u$. Here $\alpha^{i, j}$ coincides with the identification $F(R[i](j))=S \otimes_{R} R[i](j)=S[i](j)$ and the operator $\phi: F \circ A(X) \rightarrow B(X)$ is defined by the formula $\phi(s \otimes y)=s \phi_{Y}(y)$ for $s \in S$ and $y \in A(Y)$.

1.3. Examples. Below, we consider several standard examples of operations.

1.3.1. The Steenrod operation [14]. For a prime $l \neq \operatorname{char} k$, let $\mathcal{A}$ denote the category of two-sided bigraded $R$-modules, where $R=H^{*, *}(\mathrm{pt}, \mathbb{Z} / l)$ is the bigraded ring of the motivic cohomology of a point and $A(X)$ is the bigraded $R$-module of the motivic cohomology $H^{*, *}(X, \mathbb{Z} / l)$. Let $\mathcal{B}$ be the category of two-sided bigraded $S$-modules, where $S=R[[c, d]]\left[d^{-1}\right]$. Here, we have $\operatorname{deg} d=[2(l-1)](l-1), \operatorname{deg} c=[2(l-1)-1](l-1)$, $c^{2}=\tau d+\rho c, \tau \in R^{0,1}$, and $\rho \in R^{1,1}$. The theory $B$ is a flat extension of $A$, namely, $B(X)=A(X)[[c, d]]\left[d^{-1}\right]$. The theory $B(X)$ can be described as the $d$-localization of $H\left(X \times B G_{l}, \mathbb{Z} / l\right)$, where $G_{l}$ is an extension of $\mu_{l-1}$ by $\mathbb{Z} / l$. Here, geometric localization is performed by the passage to the limit in the "telescope" $\cdots \subset \mathbf{P}^{\infty-1} \subset \mathbf{P}^{\infty}$. This interpretation will not be used. For $u \in A(X)$, we put $\phi_{X}(u)=P(u)+B(u) c=$ $P^{0}(u)+B^{0}(u) c d^{-1}+P^{1}(u) d^{-1}+B^{1}(u) c d^{-2}+P^{2}(u) d^{-2}+\cdots$. For the details, see [14, p. 34], where $\phi_{X}$ is denoted by $R$. We have $B^{i}=\beta P^{i}$, and $\phi_{X}$ is multiplicative (see [14, p. 35]). Thus, we have an operation $\phi: A \rightarrow B$ (see Subsection 1.2.4).

For $l \neq 2$, this operation can be simplified and represented in the same form as the total Steenrod operation in topology, because $\tau=\rho=0$ for $l \neq 2$. Let $A$ be the same category as above, and let $B(X)=A(X)[[d]]\left[d^{-1}\right]$. For $u \in A(X)$, the simplified Steenrod operation acts by the formula $u \mapsto P(u)=P^{0}(u)+P^{1}(u) d^{-1}+P^{2}(u) d^{-2}+\ldots$ This operation is multiplicative and gives rise to an operation $A \rightarrow B$.

The Chern character, the Adams operations, and the Landweber-Novikov operation are standard examples. In the proof of the Riemann-Roch theorem given below, it is crucial that the operations are defined not only on the cohomology of varieties, but also on the cohomology of pairs (with at least smooth support). In particular, it is important that the operation is natural with respect to extension of support. To the best of my knowledge, none of the above-mentioned operations was constructed in the required generality. Therefore, in the examples below, we assume their existence.

1.3.2. Chern character. In this example, $A$ is an essentially algebraic $K$-theory. More precisely, $A(X)=K(X)[\beta]\left[\left[\beta^{-1}\right]\right]$, where $\operatorname{deg} \beta=[-2](-1)$ and $\operatorname{deg} x=[-i](0)$ for $x \in K_{i}(X)$. Thus, $A$ takes values in the category of bigraded $R$-modules with $R^{p, q}=$ $K_{2 q-p}(\mathrm{pt}) \beta^{-q}$. The theory $B$ is represented by motivic cohomology. More precisely, $B(X)=H(X, \mathbb{Q})[[\gamma]]\left[\gamma^{-1}\right]$, where $\operatorname{deg} \gamma=[-2](-1)$. Thus, $B$ takes values in the category 
of bigraded $S$-modules with $S^{p, q}=\cdots+H^{p-2, q-1}(\mathrm{pt}) \gamma^{-1}+H^{p, q}(\mathrm{pt})+H^{p+2, q+1}(\mathrm{pt}) \gamma+\cdots$ (the series are finite to the left and infinite to the right).

For a variety $X$, we have a ring homomorphism $\operatorname{ch}^{0,0}: K_{0}(X)=A^{0,0}(X) \rightarrow B^{0,0}(X)$ given on the line bundles by the formula $[L] \mapsto \exp \left(c_{1}(L) \gamma\right)$. If $\mathrm{ch}^{0,0}$ is extended to an operation ch $: K(X) \rightarrow H(X, \mathbb{Q})[[\gamma]]\left[\gamma^{-1}\right]$ on the category of all spaces (see [15]) and this extension satisfies the properties described in Subsection 1.2.4, then it gives rise to an operation $A \rightarrow B$ in the sense of Subsection 1.2.2. This operation is quasistable (see Subsection 2.5.1).

1.3.3. Adams operations. For $d \neq 0$ and a variety $X$, we have operators $\psi^{d}$ : $K_{n}(X) \rightarrow K_{n}(X)[16,1.4]$. Here $\psi^{d}(x \cdot y)=\psi^{d}(x) \cdot \psi^{d}(y)$, where $x \in K_{n}(X)$ and $y \in K_{m}(X)$ (this was proved in $[16,1.4]$ if $X$ is affine). Assuming that there exists a multiplicative extension of $\psi^{d}$ to the category of smooth open pairs, we define an operation $\phi$ for the version of $K$-theory discussed in Subsection 1.3.2 by putting $\phi(\beta)=\beta$ and $\phi=\psi^{d}$ on $K^{n, 0}$. For example, the operation $\psi^{-1}$ is induced by the functor $E \mapsto E^{\vee}$ and is defined completely.

In Subsection 2.5.3, we shall prove that if $\Lambda$ is a ring flat over $\mathbb{Z}$, then the operation $\phi \otimes \Lambda: A \otimes \Lambda \rightarrow A \otimes \Lambda$ is quasistable if and only if $d$ is invertible in $\Lambda$.

1.3.4. Landweber-Novikov operations. The topological Landweber-Novikov operation $s=\sum s_{\alpha} t^{\alpha}: M U(X) \rightarrow M U\left[\left[t_{1}, t_{2}, \ldots\right]\right](X)$, where $\alpha=\left(\alpha_{1}, \alpha_{2}, \ldots\right)$ runs over the finitary sequences in $\mathbb{N}$ and $t^{\alpha}=t_{1}^{\alpha_{1}} t_{2}^{\alpha_{2}} \cdots$, is multiplicative and satisfies

$$
s_{\alpha}[X]= \begin{cases}c_{1}(N)^{n+1} & \text { if } \alpha=(n, 0,0, \ldots), \\ 0 & \text { otherwise },\end{cases}
$$

where $X$ and $Y$ are compact complex varieties, $i: X \subset Y$ is an embedding by a divisor, $N=\mathcal{O}(X),[X] \in M U^{2}(X)$ is the class of $i$, and the $c_{j}$ are Conner-Floyd classes (see $[17$, p. 346$])$.

To the best of my knowledge, the MGL-version of the Landweber-Novikov operations has not been constructed yet. Thus, in this example, we consider a hypothetic operation $s: \mathbf{M G L} \rightarrow \mathbf{M G L}\left[\left[t_{1}, t_{2}, \ldots\right]\right]$, where $\operatorname{deg} t_{i}=[-2 i](-i)$. We assume that this operation is multiplicative and satisfies (2), where the $c_{j}$ are the Chern classes (see [10, Subsections 2.5.2 and 2.6.1]) corresponding to the tautological orientation in MGL (Subsection 2.5.4). This operation is stable.

\section{§2. Operations IN ORIENTED THEORIES}

Here, we present the main results of the paper, introduce the Jacobian of an operation and its Todd series, and prove the Riemann-Roch theorem.

2.1. Orientations, parameters, and transfers. From now on, the cohomology theories $A$ and $B$ are oriented [10,2] and $\phi: A \rightarrow B$ is an operation between them (see Subsection 1.2.2).

2.1.1. Parameters. By definition, a parameter in a theory $A$ is an element $u \in$ $A^{2,1}\left(\mathbf{P}^{\infty}\right)$ such that $u \mid \mathbf{P}^{0}=0$, and 1 and $u \mid \mathbf{P}^{1}$ form a basis of $A\left(\mathbf{P}^{1}\right)$ over $R$ (see [10, 2.4.1]). There is a bijection between the orientations and the parameters (see [10, 2.6.1]), and we assume that the orientations of $A$ and $B$ are given by parameters $u \in A^{2,1}\left(\mathbf{P}^{\infty}\right)$ and $v \in B^{2,1}\left(\mathbf{P}^{\infty}\right)$.

2.1.2. Transfers. An oriented theory is equipped automatically with transfers compatible with orientations; see [10, 3.3.1]. This means that, for every mapping $f: X \rightarrow Y$ proper on a support $S \subset X$ and for every support $T$ such that $f(S) \subset T$, we have a transfer $f_{!}=f_{!}^{S, T}: A_{S}(X) \rightarrow A_{T}(Y)$ of degree $(d)[2 d]$, where $d=\operatorname{dim} Y-\operatorname{dim} X$, 
such that the following functoriality conditions are fulfilled: $(f g)_{!}=f_{!} g_{!}$and id $!=\mathrm{id}$; linearity with respect to the base, i.e., $f_{!}^{S \cap S^{\prime}, T \cap T^{\prime}}\left(\mathrm{id} \cdot f^{*}\right)=f_{!}^{S, T} \cdot$ id as operators $A_{S}(X) \times A_{T^{\prime}}(Y) \rightarrow A_{T \cap T^{\prime}}(Y)$, where $T^{\prime}$ is an arbitrary support in $Y$ and $S^{\prime}=f^{-1}\left(T^{\prime}\right)$; and invariance under a transversal change of base, namely, for smooth varieties $X, Y$, and $\tilde{Y}$, for transversal mappings $f: X \rightarrow Y$ and $g: \tilde{Y} \rightarrow Y$, and for supports $S \subset X$ and $T \subset Y$ such that $f(S) \subset T$ and $f$ is proper on $S$, the following diagram is commutative:

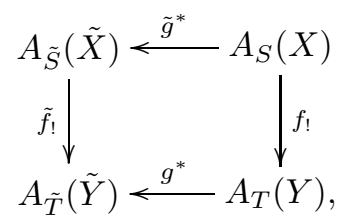

where $\tilde{f}=\tilde{Y} \times_{Y} f, \tilde{g}=g \times_{Y} X, \tilde{S}=(\tilde{g})^{-1} S$, and $\tilde{T}=(\tilde{f})^{-1} T$.

The fact that a transfer is compatible with an orientation means that

$$
u \mid \mathbf{P}^{n}=f_{!}(1)
$$

for a linear embedding $f: \mathbf{P}^{n-1} \rightarrow \mathbf{P}^{n}$.

In theories $A$ and $B$, transfers compatible with orientations are denoted by $f_{A}$ and $f_{B}$, respectively. Furthermore, if it is necessary to make the dependence on an orientation explicit, we write $f_{u}$ and $f_{v}$, respectively, where $u$ and $v$ are the orientation fixing parameters in $A$ and $B$, respectively (see Subsection 2.1.1).

2.2. The Jacobian of an operation and the Todd classes. By taking into account the identifications $A^{*, *}\left(\mathbf{P}^{\infty}\right) \simeq R[[u]]$ and $B^{*, *}\left(\mathbf{P}^{\infty}\right) \simeq S[[v]]$ (see $\left.[10,2.4 .2]\right)$, the elements $A^{*, *}\left(\mathbf{P}^{\infty}\right)$ and $B^{*, *}\left(\mathbf{P}^{\infty}\right)$ can be viewed as series.

2.2.1. Definition. The Jacobian of an operation $\phi: A \rightarrow B$ is the series

$$
j_{\phi}=\phi(u) / v=a_{0}+a_{-1} v+\cdots \in B^{0,0}\left(\mathbf{P}^{\infty}\right), \quad a_{i} \in B^{-2 i,-i}(\mathrm{pt}) .
$$

For a quasistable operation $\phi$, i.e., if $a_{0}$ is invertible, we have the Todd series $\operatorname{td}_{\phi}=j_{\phi}^{-1}$.

The reasons why the series $j_{\phi}$ is called the Jacobian of $\phi$ are indicated below in Subsection 3.2.

2.2.2. The Bernoulli classes of an operation $\phi$. The classes $b_{n} \in B^{-2 n,-n}(\mathrm{pt})$ determined by the expansion $\operatorname{td}_{\phi}(v)=b_{0}+b_{1} v+b_{2} v^{2}+\cdots$ coincide, in essence, with $B_{n} / n$ ! for $\phi=\mathrm{ch}$, where the Bernoulli numbers $B_{n}$ are given by the expansion $\operatorname{td}_{\mathrm{ch}}(t)=$ $t /\left(1-e^{-t}\right)=1+B_{1} t+B_{2} / 2 ! t^{2}+\cdots$ (see 2.5.1). The generalization of the Bernoulli numbers corresponding to the Chern character from $K$-theory to cobordism was considered in [12]. The divisibility properties of the classes $b_{n}$ play the same role as the divisibility properties of the Bernoulli numbers.

Below, we show how an arbitrary cohomology class of $\mathbf{P}^{\infty}$ determines certain characteristic classes of vector bundles. In Subsection 2.2.5, we shall apply this construction to introduce the characteristic classes related to the series $j_{\phi}$ and $\operatorname{td}_{\phi}$.

2.2.3. Multiplicative characteristic classes. Any class $\gamma \in B^{p, q}\left(\mathbf{P}^{\infty}\right)$ uniquely determines classes $\gamma(E) \in B^{p n, q n}(X)(n=\mathrm{rk} E)$ defined for all vector bundles $E / X$ and having the following properties: multiplicativity, i.e., $\gamma(E+F)=\gamma(E) \gamma(F)$; naturality, which means that $\gamma(F)=f^{*} \gamma(E)$ for each specialization $(f, \tilde{f}): E / X \rightarrow F / Y$, i.e., for 
each Cartesian square

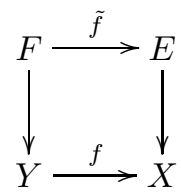

compatible with vector structures and normalized by the conditions $\gamma(0)=1$ and $\gamma\left(\mathcal{O}_{\mathbf{P} \infty}(1)\right)=\gamma$. We note that, in general, the relation $\gamma(1)=0$, where 1 is the trivial line bundle, may fail.

Furthermore, for classes satisfying the above properties, the property of extended multiplicativity is valid, i.e., $\gamma(E)=\gamma(F) \gamma(Q)$ for every exact sequence of vector bundles $0 \rightarrow F \rightarrow E \rightarrow Q \rightarrow 0$.

Existence and uniqueness follow from normalization, naturality, the homotopy invariance of the theory $B$, the splitting principle (see [10, Subsection 2.4.4]), and the existence of a characteristic operator $[10$, Subsection 4.4.3] for line bundles. We explain the statement concerning the characteristic operator. Though, in general, a line bundle $L / X$ is not obtained from the tautological bundle by the mapping $i_{L}: X \rightarrow \mathbf{P}^{\infty}$, we have the corresponding operator $i_{L}^{*}: A\left(\mathbf{P}^{\infty}\right) \rightarrow A(X)$ called characteristic and determined by the fact that $i_{\tau}^{*}=\mathrm{id}$ for the tautological bundle $\tau / \mathbf{P}^{\infty}$ and $i_{M}^{*}=f^{*} i_{L}^{*}$ for each specialization $(f, \tilde{f}): L / X \rightarrow M / Y$.

Extended multiplicativity is a consequence of the fact that the embeddings and epimorphisms of vector bundles are homotopy split [20]; more precisely, for each embedding $i: E \rightarrow F$ (epimorphism $p: F \rightarrow E$ ) of vector bundles on $X$ there is an affine bundle $f: \tilde{X} \rightarrow X$ for which $f^{*} i$ (respectively, $f^{*} p$ ) is split, where the affine bundle is a torsor of a vector bundle.

2.2.4. The Jacobi classes. For each smooth variety $X$ and each vector bundle $E / X$, the Jacobian $j_{\phi}$ of an operation $\phi$ determines (see Subsection 2.2.3) the Jacobi class $j_{\phi}(E) \in B^{0,0}(X)$. For example, $j_{\phi}\left(1^{n}\right)=a_{0}^{n}$, where $a_{0}$ is the free term of $j_{\phi}$ (see Subsection 2.2.1)

2.2.5. The Todd classes. Here, the operation $\phi$ is quasistable, i.e., $a_{0}$ is invertible. For each smooth variety $X$ and each vector bundle $E / X$, the Todd series $\operatorname{td}_{\phi}$ of $\phi$ yields (see Subsection 2.2.3) the Todd class $\operatorname{td}_{\phi}(E) \in B^{0,0}(X)$. For example, $\operatorname{td}\left(1^{n}\right)=a_{0}^{-n}$, where $a_{0}$ is the free term of $j_{\phi}$ (see Subsection 2.2.1).

We define the Todd class of a mapping $f: X \rightarrow Y$ of smooth varieties by the formula

$$
\operatorname{td}_{\phi}(f)=\operatorname{td}_{\phi}\left(T_{X}\right) / f^{*} \operatorname{td}_{\phi}\left(T_{Y}\right)=f^{*} j_{\phi}\left(T_{Y}\right) / j_{\phi}\left(T_{X}\right) \in B^{0,0}(X),
$$

where $T_{X}$ and $T_{Y}$ are tangent bundles of $X$ and $Y$. If $f$ is an embedding, then

$$
\operatorname{td}_{\phi}(f)=j_{\phi}\left(N_{X} Y\right) \in B^{0,0}(X),
$$

where $N_{X} Y$ is the normal to $X$ at $Y$. This follows from the exact sequence $0 \rightarrow T_{X} \rightarrow$ $T_{Y} \rightarrow N_{X} Y \rightarrow 0$ and extended multiplicativity (Subsection 2.2.3). The Todd class of a smooth mapping $f: X \rightarrow Y$ can be calculated by the formula $\operatorname{td}_{\phi}(f)=\operatorname{td}_{\phi}\left(T_{X / Y}\right)$, where $T_{X / Y}$ is the relative tangent bundle. This is a consequence of the fact that the sequence $0 \rightarrow T_{X / Y} \rightarrow T_{X} \rightarrow f^{*} T_{Y} \rightarrow 0$ is exact.

The Todd class of a smooth variety $X$ is defined by the formula $\operatorname{td}_{\phi}(X)=\operatorname{td}_{\phi}(p)$, where $p$ is the structure projection $X \rightarrow$ pt. In other words,

$$
\operatorname{td}_{\phi}(X)=\operatorname{td}_{\phi}\left(T_{X}\right) \in B^{0,0}(X) .
$$


For example, the extended multiplicativity (see Subsection 2.2.3) and the exact sequence $0 \rightarrow \mathcal{O}_{\mathbf{P}^{n}} \rightarrow \mathcal{O}_{\mathbf{P}^{n}}(1)^{n+1} \rightarrow T_{\mathbf{P}^{n}} \rightarrow 0$ imply the relation

$$
\operatorname{td}_{\phi}\left(\mathbf{P}^{n}\right)=a_{0} \operatorname{td}_{\phi}^{n+1}(v) \mid \mathbf{P}^{n} .
$$

If we define the Todd class of a virtual bundle $(-E)$ as $\operatorname{td}_{\phi}^{-1}(E)$, then $\operatorname{td}_{\phi}(X)=$ $j_{\phi}\left(-T_{X}\right)$, where $\left(-T_{X}\right)$ is regarded as a "virtual normal bundle" to $X$ of negative rank $-\operatorname{dim} X$. For a stable operation, we have $a_{0}=1$, and the rank is immaterial. In the general case, the rank affects the Todd class.

2.3. Operations and differentials. Below, we present some information concerning interaction of an operation with differential forms and their residues. This information will be used in the proof of the Riemann-Roch theorem.

2.3.1. The comparison functor and differentials. For a commutative monoid $E \in A$ and a commutative $E$-algebra $D$, the module of differentials $\Omega_{E} D$ is defined as the $D$ module of values of the universal $E$-linear differentiation $d: D \rightarrow \Omega_{E} D$ (if it exists); see $[10$, Subsection 4.5.3].

In what follows, we use the case where $E=A(Y)$ for a smooth variety $Y, D=\tilde{A}(Y \times$ $\left.\mathbf{P}^{\infty}\right)$ is the localization of $A\left(Y \times \mathbf{P}^{\infty}\right)$ with respect to affine parameters (see [10, Subsection 4.5.2]), and $A$ is an oriented theory. In this case, $\Omega_{E} D$ exists [10, Subsection 4.5.3], and since $D / E$ is free [10, Subsection 4.5.2], the module of differentials $\Omega_{F E} F D \in \mathcal{B}$ also exists. Furthermore, since $F$ is monoidal, the operator $F(d): F D \rightarrow F\left(\Omega_{E} D\right)$ is an $F E$-linear differentiation. By universality, this differentiation uniquely determines an operator $\alpha: \Omega_{F E} F D \rightarrow F\left(\Omega_{E} D\right)$, which is an isomorphism. This isomorphism allows us to identify $F\left(\Omega_{E} D\right)$ with $\Omega_{F E} F D$. Moreover, in the case in question, we have (see [10, Subsection 4.5]) the residue operator

$$
\operatorname{res}_{M}: \Omega_{M} D \rightarrow M \otimes C_{A}^{-1},
$$

where $M=A_{T}(Y), T$ is a support in $Y$, and $\Omega_{M} D=M \otimes_{E} \Omega_{E} D$ (see [10, Subsection 4.5.3]). Accordingly, we have the residue operator $\operatorname{res}_{F M}: \Omega_{F M} F D \rightarrow F M \otimes C_{B}^{-1}$; taking the identification $F\left(\Omega_{E} D\right) \simeq \Omega_{F E} F D$ into account, we obtain

$$
\tilde{F}\left(\operatorname{res}_{M}\right)=\operatorname{res}_{F M},
$$

where $\tilde{F}\left(\operatorname{res}_{M}\right)$ is the operator defined in Subsection 1.2.1. This follows from the explicit formula for $\operatorname{res}_{u}$ written in [10, Subsection 4.5.4] and the fact that the residue can be calculated if we know the parameter, i.e., res $=\operatorname{res}_{u}$.

2.3.2. The action of an operation on differentials. Since $\phi$ is multiplicative, the operator $d_{B} \circ \phi: F D \rightarrow \Omega_{K} H$, where $H=\tilde{B}\left(Y \times \mathbf{P}^{\infty}\right)$ is the localization of $B\left(Y \times \mathbf{P}^{\infty}\right)$ with respect to affine parameters (see [10, Subsection 4.5.2]), $K=B(Y)$, and $d_{B}: H \rightarrow$ $\Omega_{K} H$ is the universal differentiation, is an $F E$-linear differentiation, where the $F E$ module structure on $H$ is given by the operator $\phi: F E \rightarrow K$ and the natural $K$-module structure on $H$. By the universality of $F\left(d_{A}\right)$, where $d_{A}: D \rightarrow \Omega_{E} D$ is the universal differentiation, we have a unique $F E$-linear operator

$$
\phi_{\Omega}: \Omega_{F E} F D \rightarrow \Omega_{K} H
$$

such that $\phi\left(F\left(d_{A}\right)\right)=d_{B} \circ \phi_{\Omega}$.

Moreover, we have an action of $\phi$ on differential forms, i.e., an operator $\phi:\left(\Omega_{E} D\right)^{0,0} \rightarrow$ $\left(\Omega_{K} H\right)^{0,0}$. Specifically, for $\omega \in\left(\Omega_{E} D\right)^{0,0}=\operatorname{Hom}\left(R, \Omega_{E} D\right)$, the form $\phi(\omega)$ is represented by the composition $S \stackrel{F \omega}{\longrightarrow} \Omega_{F E} F D \stackrel{\phi_{\Omega}}{\longrightarrow} \Omega_{K} H$ if we use the identifications $S \simeq F(R)$ and $F\left(\Omega_{E} D\right) \simeq \Omega_{F E} F D($ see 2.3 .1$)$. 
If the operation $\phi: A \rightarrow B$ is quasistable, i.e., $\phi(u)$ is the parameter of $B\left(\mathbf{P}^{\infty}\right)$, then

$$
\phi \circ \operatorname{res}_{F M} \tilde{F}(\omega)=\operatorname{res}_{N} \phi(\omega),
$$

where $N=B_{T}(Y)$. This follows from the explicit formula for $\operatorname{res}_{F M}=\operatorname{res}_{\phi u}$; see [10, Subsection 4.5.4].

2.3.3. Operations and invariant forms. Let $\omega_{u} \in\left(\Omega_{E} D\right)^{0,0}$ and $\omega_{v} \in\left(\Omega_{K} H\right)^{0,0}$ be the normalized invariant differential forms related to the fixed orientations of $A$ and $B$ (see [10, Subsection 2.7.4]). Then

$$
\phi\left(\omega_{u}\right)=a_{0} \omega_{v},
$$

where $a_{0}$ is the free term of $j_{\phi}$ (see Subsection 2.2.1). Indeed, since the operation $\phi$ induces a homomorphism of formal groups, it preserves the invariance of the form. Therefore, to prove (10) it suffices to calculate the coefficient of $d v$.

2.4. The Riemann-Roch theorem. As above, let $A$ and $B$ be oriented theories, and let $\phi: A \rightarrow B$ be an operation.

The following theorem is the Riemann-Roch theorem for embeddings. This theorem is one of the main results of the paper. Unlike the general Riemann-Roch theorem (see Subsection 2.4.3 below), this theorem does not require the quasistability of $\phi$.

2.4.1. Theorem. Let $f: X \rightarrow Y$ be an embedding of smooth varieties proper on a support $S \subset X$ such that $f(S) \subset T$, where $T$ is a support in $Y$. Then

$$
\phi \circ \tilde{F}\left(f_{A}\right)=f_{B} \circ\left[j_{\phi}\left(N_{X} Y\right) \cdot \phi\right] .
$$

In other words, the following diagram is commutative:

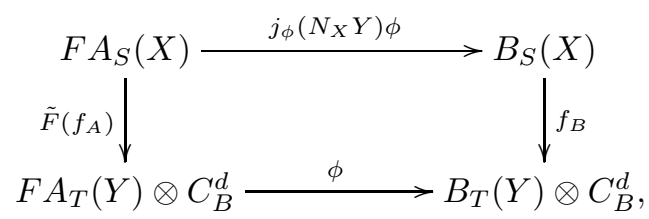

where $d=\operatorname{dim} X-\operatorname{dim} Y, F$ is the comparison functor for target categories that corresponds to the operation (see Subsections 1.2 .1 and 1.2.2), $\tilde{F}\left(f_{A}\right)$ is the operator defined in Subsection 1.2.1, and $\phi$ in the second line acts via the first argument.

Proof. The theorem is proved in several steps. We put $N=N_{X} Y$.

Step 1. Let $X=\mathbf{P}^{n-1}, Y=\mathbf{P}^{n}$. Let $f$ be a linear embedding, and let $S=X$ and $T=Y$. Since the restriction $f^{A}: A\left(\mathbf{P}^{n}\right) \rightarrow A\left(\mathbf{P}^{n-1}\right)$ is a split epimorphism because $A$ is orientable [10, Subsection 2.5.9], it suffices to prove that the compositions of $f^{A}$ with the two sides of (11) coincide. Here, the composition $f_{A} f^{A}: A\left(\mathbf{P}^{n}\right) \rightarrow A\left(\mathbf{P}^{n}\right)$ coincides with multiplication by $u \mid \mathbf{P}^{n}$ (see (3) and [10, Subsection 3.2.2]). Thus, $\phi \circ f_{A} f^{A}$ coincides with multiplication by $\phi(u) \mid \mathbf{P}^{n}$. On the other hand, we have

$$
f_{B} \circ\left[j_{\phi}(N) \cdot \phi \circ F\left(f^{A}\right)\right]=j_{\phi}(\mathcal{O}(1)) \cdot f_{B} \circ\left[\phi \circ F\left(f^{A}\right)\right]=j_{\phi}(\mathcal{O}(1)) \cdot f_{B} f^{B},
$$

where the first identity in (13) follows from linearity with respect to the base (see Subsection 2.1.2), because $N$ is the restriction of $\mathcal{O}(1)$ to $\mathbf{P}^{n-1}$. The second identity in (13) follows from the fact that $\phi$ is functorial (see Subsection 1.2.2). Since $f_{B} f^{B}$ coincides with multiplication by $v \mid \mathbf{P}^{n}$ (like for the theory $A$, see above), we see that (11) reduces to the formula $\phi(u)=j_{\phi}(\tilde{N}) \cdot v$, which is valid by the normalization property of the classes $j_{\phi}$ (see Subsection 2.2.3). 
Step 2. Let $X=\mathbf{P}^{n}, Y=\mathbf{P}^{n+1}$. Let $f$ be a linear embedding, and let $S=X$ and $T=Y$. In this case, relation (11) reduces to the preceding case if we recall that (a) $f_{A}^{X, Y}=i_{A}^{X, Y} \circ f_{A}^{X, X}$, where $i: \mathbf{P}^{n+1} \rightarrow \mathbf{P}^{n+1}$ is the identity mapping (this follows from the fact that the transfers are functorial, see Subsection 2.1.2); (b) the transfer $i_{A}^{X, Y}$ coincides with the operator of extension of supports $A_{X}\left(\mathbf{P}^{n+1}\right) \rightarrow A\left(\mathbf{P}^{n+1}\right)[10$, Subsection 2.3.1] and commutes with $\phi$ by the functoriality of the operation, because the operator of extension of supports is induced by a mapping of pairs, i.e., by a morphism of the category of spaces $\mathcal{S}$; (c) $i_{A}^{X, Y}$ is a monomorphism (see [10, Subsection 1.3.15]).

Step 3. Suppose $E$ is a bundle on $X, Y=E, f$ is the zero section, and $S=T=X$. In this case, the splitting principle $[10,2.4 .4]$ and the fact that the Thom operator is multiplicative (see [10,2.2.1]) allow us to reduce relation (11) to the case where $E$ is a line bundle. The case of a line bundle reduces to the case where $E=\mathcal{O}(1) / \mathbf{P}^{n}$ because the line bundle $\mathcal{O}(1) / \mathbf{P}^{\infty}[20]$ is homotopy universal. Now, (11) follows from the preceding step because $A_{X}(E)=A_{\mathbf{P}^{n}}\left(\mathbf{P}^{n+1}\right)$ by excision.

Step 4. Suppose $E$ is a bundle on $X, Y=E, f$ is the zero section, and $T=S$. In this case, relation (11) reduces to the case analyzed above because $f_{A}^{S, S}=f_{A}^{X, X}(1) \cdot p_{S}^{A}$ by the projection formula (i.e., by linearity with respect to the base, see Subsection 2.1.2); here the mapping of pairs $p_{S}:\left(E, E-p^{-1} S\right) \rightarrow(X, X-S)$ is induced by the structure projection $p: E \rightarrow X$.

Step 5 . Let $T=S$. In this case, the theorem follows from the fact that diagram (12) is isomorphic to a similar diagram for the zero section $z: X \rightarrow N$,

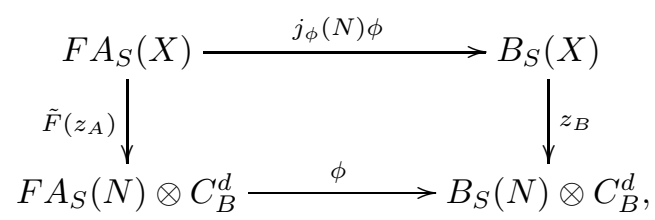

which is commutative by the preceding step. To compare diagrams (12) and (14), we use deformation to the normal; more precisely, we use the diagram

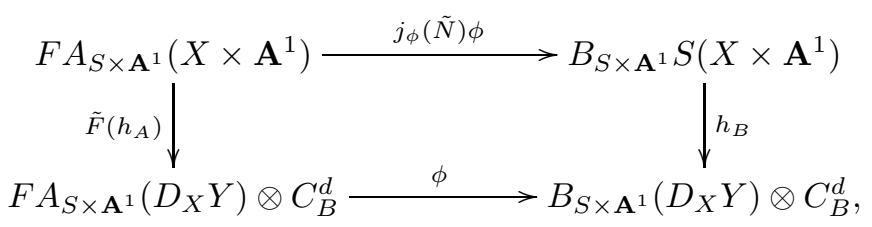

where $D_{X} Y$ is deformation to the normal (see [10, Subsection 4.2.3]), $h=D f: X \times \mathbf{A}^{1}=$ $D_{X} X \rightarrow D_{X} Y, \tilde{N}$ is the normal to $X \times \mathbf{A}^{1}$ at $D_{X} Y$, and $S \times \mathbf{A}^{1}$ is embedded in $D_{X} Y$ by $h$.

The special fiber $s$ and a typical fiber $g$ of deformation to the normal (see [10, Subsection 4.2.3]) induce operators from the vertices of (15) to the corresponding vertices of the diagrams (14) and (12); these operators are morphisms of diagrams. This is proved by verifying that certain squares are commutative. The commutativity of the squares related to the horizontal arrows follows from the functoriality of $\phi$ and isomorphisms $s^{*} \tilde{N} \simeq g^{*} \tilde{N} \simeq N$. The commutativity of the squares related to the vertical arrows follows because the corresponding squares are transversal at the geometric level and the transfers commute with a transversal change of the base (see Subsection 2.1.2). The geometric squares are transversal because the deformation $D_{X} Y \rightarrow \mathbf{A}^{1}$ (see [10, Subsection 4.2.4]) is smooth. 
Since the above morphisms of diagram (15) to diagrams (14) and (12) are isomorphisms by [10, Subsection 1.3.7], this proves that diagrams (12) and (14) are isomorphic.

Step 6 . In the general case, the proof of (11) reduces to the case analyzed at the preceding step because $f_{!}^{S, T}=i_{!}^{S, T} \circ f_{!}^{S, S}$ by the functoriality of transfers (see Subsection 2.1.2), where $i$ is the identity mapping of $Y$. Diagram (12) for $i$ is commutative by the functoriality of $\phi$, because the transfer $i_{!}^{S, T}$ is induced by a mapping of open pairs and coincides with the operator of extension of supports from $S$ to $T$; see [10, Subsection 2.3.1].

2.4.2. Corollary. Under the conditions of Subsection 2.4.1, assume in addition that $A$ and $B$ take values in categories of bigraded modules, the operation $\phi$ is constructed as in Subsection 1.2.4, and $x \in A_{S}^{p, q}(X)$. Then formula (11) simplifies, taking the form

$$
\phi\left(f_{A}(x)\right)=f_{B}\left(j_{\phi}\left(N_{X} Y\right) \cdot \phi(x)\right) .
$$

The theorem below is the general Riemann-Roch theorem, which is the main result of the present paper. As above, let $A$ and $B$ be oriented theories, and let $\phi: A \rightarrow B$ be an operation. Also, we assume that $\phi$ is quasistable (see Subsection 1.2.2); i.e., the free term $a_{0}$ of the series $j_{\phi}$ (see Subsection 2.2.1) is invertible.

2.4.3. Theorem. Let $f: X \rightarrow Y$ be a mapping of smooth varieties proper on a support $S \subset X$, let $T$ be a support in $Y$, and let $f(S) \subset T$. Then the following diagram is commutative:

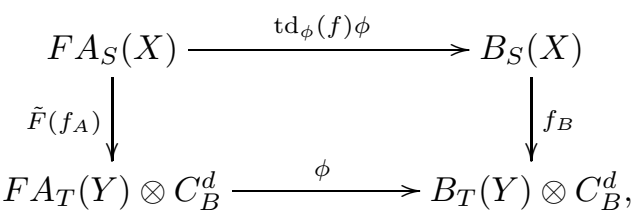

where $d=\operatorname{dim} X-\operatorname{dim} Y, F$ is the comparison functor for target categories that corresponds to the operation (see Subsections 1.2.1 and 1.2.2), $\tilde{F}\left(f_{A}\right)$ is the operator defined in Subsection 1.2.1, and $\phi$ in the second line acts via the first argument.

Proof. Since $X$ is quasiprojective, we have an embedding in $: X \rightarrow \mathbf{P}^{n}$, and the mapping $i=f \times i n: X \rightarrow Y \times \mathbf{P}^{n}$ is proper on $S$ because the graph of $i n$ is closed. Since $f=p \circ i$, where $p$ is the projection $Y \times \mathbf{P}^{n} \rightarrow Y$ proper on the support $p^{-1} T \supset i(S)$, and for the embedding $i$ the claim is proved in Subsection 2.4.1, we see that it suffices to prove the theorem for a projection $p$ and verify that the commutativity of diagram (17) for $f=g h$ follows from the commutativity of the corresponding diagrams for $f=g$ and $f=h$. The second statement is a consequence of the relation $\operatorname{td}_{\phi}(g h)=h^{*}\left[\operatorname{td}_{\phi}(g)\right] \operatorname{td}_{\phi}(h)$, which follows directly from the definition (4) in Subsection 2.2.5. The first statement follows from the commutativity of the diagram

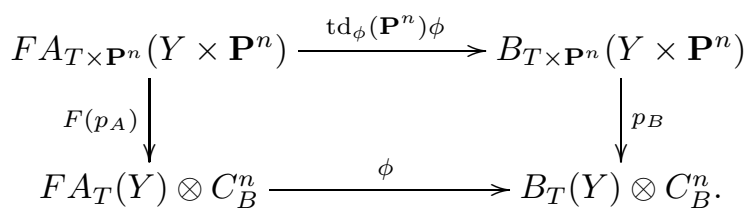

Consider the embedding $z: Y \times \mathbf{P}^{n} \rightarrow Y \times \mathbf{P}^{\infty}$ induced by the linear embedding $\mathbf{P}^{n} \rightarrow \mathbf{P}^{\infty}$. Since $\operatorname{td}_{\phi}\left(\mathbf{P}^{n}\right)=z^{B}\left[a_{0} \operatorname{td}_{\phi}^{n+1}(v) \mid \mathbf{P}^{n}\right]$ (see (6) in Subsection 2.2.5), the 
following diagram is commutative by the functoriality of $\phi$ :

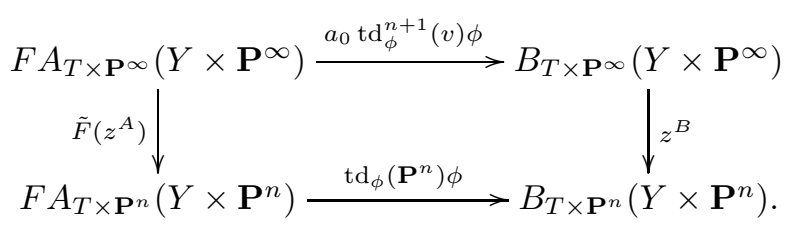

Since the restriction $z^{A}: A_{T \times \mathbf{P} \infty}\left(Y \times \mathbf{P}^{\infty}\right) \rightarrow A_{T \times \mathbf{P}^{n}}\left(Y \times \mathbf{P}^{n}\right)$ is a split epimorphism by [10, Subsection 2.5.12], the theorem follows from the fact that the diagram

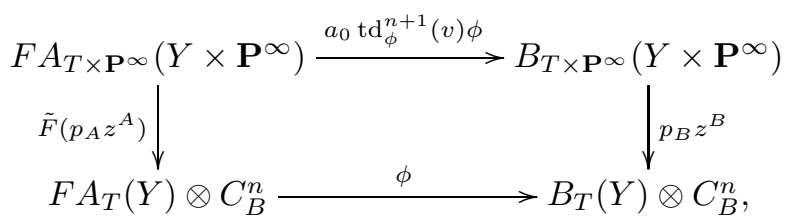

which is the composition of diagrams (19) and (18), is commutative. To prove this, we use Quillen's formula (see [10, Subsection 3.2.6])

$$
p_{A} z^{A}=\operatorname{res}_{M} \frac{\omega_{u}}{u^{n+1}}, \quad p_{B} z^{B}=\operatorname{res}_{N} \frac{\omega_{v}}{v^{n+1}},
$$

where $\omega_{u}$ and $\omega_{v}$ are normalized invariant differential forms for $A$ and $B$ (see Subsection 2.3.3) and $\operatorname{res}_{M}: \Omega_{M} D \rightarrow M \otimes C_{A}^{-1}$ and $\operatorname{res}_{N}: \Omega_{N} H \rightarrow N \otimes C_{B}^{-1}$ are residue operators [10, Subsection 4.5.4]. Here, $M=A_{T}(Y), N=B_{T}(Y)$, and $D$ and $H$ are localizations of $A\left(Y \times \mathbf{P}^{\infty}\right)$ and $B\left(Y \times \mathbf{P}^{\infty}\right)$ with respect to affine parameters (see Subsections 2.3.1 and 2.3.2). Using the identification $M \otimes_{E} A\left(Y \times \mathbf{P}^{\infty}\right) \simeq A_{T \times \mathbf{P}}\left(Y \times \mathbf{P}^{\infty}\right)$ (see [10, Subsection 2.4.3]), we see that the operator $\operatorname{res}_{M}\left(\omega_{u} / u^{n+1}\right)$ is the composition

$$
M \otimes_{E} A\left(Y \times \mathbf{P}^{\infty}\right) \stackrel{u^{-n-1}}{\longrightarrow} M \otimes_{E} D \otimes C_{A}^{n+1} \stackrel{\omega_{u}}{\longrightarrow} \Omega_{M} D \otimes C_{A}^{n+1} \stackrel{\text { res }}{\longrightarrow} M \otimes C_{A}^{n} .
$$

By (21), the path "down and right" in diagram (20), i.e., $\phi \tilde{F}\left(p_{A} z^{A}\right)$, is equal to

$$
\begin{aligned}
& \phi \tilde{F} \\
& \left.\quad=\operatorname{res}_{M} \frac{\omega_{u}}{u^{n+1}}\right)=\phi \circ \operatorname{res}_{F M} \frac{\tilde{F}\left(\omega_{u}\right)}{\tilde{\tilde{F}}(u)^{n+1}}=\operatorname{res}_{N} \frac{\omega_{v}}{v^{n+1}} \circ \frac{a_{0} v^{n+1}}{\phi(u)^{n+1}} \phi,
\end{aligned}
$$

where the first identity follows from (7) in Subsection 2.3.1, the second follows from (9) in Subsection 2.3.2, and the third follows from (10) in Subsection 2.3.3. Using the second identity in (21) and the definition of the Todd series (see Subsection 2.2.1), we deduce that diagram (20) is commutative, which completes the proof of the theorem.

An essential point in the above proof is the existence of the residue res, which is equivalent to the invariance of the residue $\operatorname{res}_{u}$ under the change of a parameter (in principle, the proof of formula (22) is based on this invariance). Here, it suffices to have invariance under the change of a local parameter.

In [10, Subsection 4.5.4], we proved the invariance of the residue for a wider class of changes of variables, namely, for a change of affine parameters (an affine parameter differs from a local one in that it admits a shift of the zero in the formal group by a nilpotent). Here, the invariance has a simple geometric meaning: the residue of a form at a point is represented by an integral along a small contour around this point, and a shift of the point by a quantity infinitesimally small relative to the formal parameter does not move the point out of the small contour, thus preserving the residue. Using this invariance, we can prove the Riemann-Roch theorem directly for a projectivization 
of a vector bundle (in the proof of Theorem 2.4.3 the trivial bundle was used), which can be useful in a more general situation, for example, when studying relatively projective varieties.

We present several corollaries to Theorem 2.4.3. The first of them, despite the triviality of the operation in question, clarifies the meaning of the Riemann-Roch theorem.

2.4.4. Corollary. Under the assumptions of Theorem 2.4.3, take $A=B$ and $\phi=\mathrm{id}$. Then the Riemann-Roch theorem simplifies, taking the form

$$
f_{u}=f_{v}\left(\operatorname{td}_{\phi}(f) \cdot \mathrm{id}\right) .
$$

This corollary makes it possible to calculate the change of a transfer under a change of a parameter. An example of such a situation will be considered below in Subsection 2.5.5.

2.4.5. Corollary. Under the conditions of Theorem 2.4.3, assume that $A$ and $B$ take values in categories of bigraded modules, the operation $\phi$ is constructed as in Subsection 1.2.4, and $x \in A_{S}^{p, q}(X)$. Then the Riemann-Roch theorem simplifies, taking the form

$$
\phi\left(f_{A}(x)\right)=f_{B}\left(\operatorname{td}_{\phi}(f) \cdot \phi(x)\right) .
$$

2.4.6. Corollary. Under the conditions of Corollary 2.4.5, assume that $\phi(u)=v$. Then the operation $\phi$ commutes with transfers, i.e.,

$$
\phi\left(f_{A}(x)\right)=f_{B}(\phi(x)) .
$$

2.5. Examples. We consider several examples illustrating the Riemann-Roch theorem.

2.5.1. The Chern character. This example is a continuation of Subsection 1.3.2. The orienting parameter is defined by the formula $u \mid \mathbf{P}^{n}=\beta^{-1}([1]-[\mathcal{O}(-1)])$; see $[10$, Subsection 3.3.2]. The orienting parameter $t \in H^{2,1}\left(\mathbf{P}^{\infty}, \mathbb{Q}\right)[[\gamma]]\left[\gamma^{-1}\right]$ is induced by the class $\mathcal{O}(1)$ under the composition Pic $\mathbf{P}^{\infty} \rightarrow H^{2,1}\left(\mathbf{P}^{\infty}, \mathbf{Z}\right.$ ) (see [5, Subsection 3.2.1] and [10, Subsection 2.6.4]). Thus, $\phi(u)=\operatorname{ch}^{2,1}\left(\beta^{-1}([1]-[\mathcal{O}(-1)])\right)=\operatorname{ch}^{0,0}([1]-[\mathcal{O}(-1)]) \gamma^{-1}=$ $\left(1-e^{-\gamma t}\right) \gamma^{-1}$. Therefore, $j_{\phi}(t)=\left(1-e^{-\gamma t}\right) /(\gamma t)$,

$$
\operatorname{td}_{\phi}(t)=\frac{\gamma t}{1-e^{-\gamma t}},
$$

and Theorem 2.4.3 transforms into a motivic version of the Riemann-Roch-Grothendieck theorem [2].

2.5.2. The Steenrod operation. This example is a continuation of Subsection 1.3.1. The orienting parameter $t$ is induced by the class $\mathcal{O}(1)$ under the composition $\operatorname{Pic} \mathbf{P}^{\infty} \rightarrow$ $H^{2,1}\left(\mathbf{P}^{\infty}, \mathbf{Z}\right) \rightarrow H^{2,1}\left(\mathbf{P}^{\infty}, \mathbf{Z} / l\right)$ (see [5, Subsection 3.2.1] and [10, Subsection 2.6.4]). Then $\phi(t)=t+t^{l} / d$,

$$
j_{\phi}(t)=1+t^{l-1} / d, \quad \text { and } \quad \operatorname{td}_{\phi}(t)=1-t^{l-1} / d+t^{2(l-1)} / d^{2}-\cdots .
$$

This follows from the definition of $\phi$ and the relations $P^{0}(t)=t[14,9.5], P^{1}(t)=t^{l}[14$, Subsection 9.8], and $P^{i}(t)=0$ for $i \geq 2$ [14, Subsection 9.9], and $\beta t^{i}=0$ (because $t$ comes from $\mathbf{Z}$ ).

For example, $j_{\phi}(E)=\left(1+\gamma_{1}^{l-1} / d\right) \cdots\left(1+\gamma_{n}^{l-1} / d\right)$ for the virtual expansion $E=$ $L_{1}+\cdots+L_{n}$ of a vector bundle. In particular, $j_{\phi}(E)=1+c_{1}(E) d^{-1}+\cdots+c_{n}(E) d^{-n}$ essentially coincides with the total Chern class for $l=2$.

As in topology (see $[18, \S \S 3,5]$ ), the Riemann-Roch theorem implies integrality theorems, i.e., properties of some characteristic classes. For example (see [11, Subsections 2.6.6, 2.6.7]), for a smooth projective variety $X$ and $[X]=p_{!}(1) \in H^{-2 n,-n}(\mathrm{pt})$, where $\operatorname{dim} X=n$ and $p: X \rightarrow$ pt is the structure morphism, we have $p_{!}\left(\operatorname{td}_{\phi}(X)\right)=\phi[X]$. Since $H^{-2 n,-n}(\mathrm{pt})=0$ for $n>0$ (by construction, there is no cohomology of negative 
weight), we obtain $[X]=0$, whence $p_{!}\left(\operatorname{td}_{\phi}(X)\right)=0$. Here $\operatorname{td}_{\phi}(X)=\operatorname{td}_{\phi}\left(T_{X}\right)=(1+$ $\left.\gamma_{1}^{l-1} d^{-1}\right) \cdots\left(1+\gamma_{n}^{l-1} d^{-1}\right)$, where $\gamma_{i}=c_{1}\left(L_{i}\right)$ for the formal expansion $T_{X}=L_{1}+\cdots+L_{n}$. Thus, for example, we have $p_{!}\left(\gamma_{1}^{l-1}+\cdots+\gamma_{n}^{l-1}\right)=0$. Since the symmetric polynomials $\left(\gamma_{1}, \ldots, \gamma_{n}\right)$ are polynomials in the Chern classes $c_{i}\left(T_{X}\right)$, we see that the transfers of these polynomials are zero. In particular, for a class in $H^{2 n, n}(X)$ we obtain the relation $H^{0,0}(\mathrm{pt})=\mathbb{Z} / l$, i.e., a congruence for the characteristic number. For instance, we have $c_{1}^{2}+c_{2}=0 \bmod 3$ for $\operatorname{dim} X=2$. In another example, we have $s_{n}(X)=0 \bmod 2$, where $s_{n}$ is the Segre class; see [19, Subsection 3.2].

2.5.3. Adams operations. This is a continuation of Subsection 1.3.3; the orientation is given in Subsection 2.5.1. Thus, $\phi(u)=\phi\left(\beta^{-1}([1]-[\mathcal{O}(-1)])\right)=\beta^{-1}(1-[\mathcal{O}(-d)])=$ $\beta^{-1}\left(1-x^{d}\right)$, where $x=1-\beta u=[\mathcal{O}(-1)] \in K_{0}\left(\mathbf{P}^{\infty}\right)$. We have $u=\beta^{-1}(1-x)$ and

$$
j_{\phi}(u)=\left(1-x^{d}\right) /(1-x)
$$

Therefore, $a_{0}=d$ (see Subsection 2.2.1), and $\phi \otimes \Lambda$ (see Subsection 1.3.3) is quasistable for $d$ invertible in $\Lambda$. In this case, the Riemann-Roch theorem coincides essentially with the theorem in [16, Subsection 7.2].

For $d=-1$ and a smooth projective variety $X$, we have $\operatorname{td}_{\phi}\left(T_{X}\right)=(-1)^{n}\left[L_{1}\right] \cdots\left[L_{n}\right]$ for the virtual expansion $T_{X}=L_{1}+\cdots+L_{n}$, whence $\operatorname{td}_{\phi}\left(T_{X}\right)=(-1)^{n}[K]^{-1}$, where $K=\Lambda^{n} T_{X}^{\vee}$ is the canonical class of $X$. Corollary 2.4.5 for the projection $f: X \rightarrow \mathrm{pt}$ reduces to the formula $\chi(E)=(-1)^{n} \chi\left(E^{\vee} \otimes K\right)$, which follows from the Serre duality.

2.5.4. The Landweber-Novikov operation. This example is a continuation of Subsection 1.3.4. Let $u$ be the parameter corresponding to the tautological orientation of MGL [10, Subsection 2.2.10]. The same parameter determines the orientation of MGL $\left[\left[t_{1}, t_{2}, \ldots\right]\right]$. Applying (2) to the linear embedding $i: \mathbf{P}^{n-1} \subset \mathbf{P}^{n}$ and using the fact that $i_{!}(1)=u \mid \mathbf{P}^{n}$ (see (3)), we obtain $\phi(u)=u+t_{1} u^{2}+t_{2} u^{3}+\cdots$ and $j_{\phi}(u)=1+t_{1} u+t_{2} u^{2}+\cdots$.

Let $f: X \rightarrow Y$ be a mapping of smooth projective varieties, and let $[f]=f_{!}\left(1_{X}\right) \in$ MGL ${ }^{-2 d,-d}(Y)$, where $d=\operatorname{dim} X-\operatorname{dim} Y$. Corollary 2.4.5 to the Riemann-Roch theorem allows us to calculate the action of the operation on the class $[f]$, namely, $s_{\alpha}[f]=f_{!}\left(c_{1}^{\alpha_{1}} c_{2}^{\alpha_{2}} \cdots\right)$, where the $c_{i}$ are Chern classes of the virtual bundle $f^{*} T_{Y}-T_{X}$.

2.5.5. Nonstandard orientation of $K$-theory. In this example, $A$ and $B$ coincide with the version of $K$-theory described in Subsection 1.3.2, the operation $\phi$ is the identity, but the orientations of $A$ and $B$ are distinct. The orientation of $B$ was given in Subsection 2.5.1; for this orientation we have $v=\beta^{-1}\left([1]-\left[L^{-1}\right]\right)$, where $L=\mathcal{O}(1) / \mathbf{P}^{\infty}$; the orientation of $A$ is given by the parameter $u=\beta^{-1}\left[L^{s}\right]\left(1-\left[L^{-1}\right]\right)$, where $s \in \mathbb{Z}$. Then $j_{\phi}=x^{s}$, where $x=[L]$, and the Riemann-Roch theorem applied to the projection $f: X \rightarrow$ pt of a smooth variety $X$ reduces to the formula

$$
f_{u}[E]=\chi\left(E \otimes K_{X}^{s}\right)
$$

where $E / X$ is a vector bundle and $K_{X}$ is the canonical class of $X$.

\section{§3. TRANSFERS AND INTEGRALS}

In the present section, our goal is to clarify the analogy between transfers and integration and, in particular, between the Riemann-Roch theorem and change of variables in integrals. Furthermore, we show that the formula for the Todd classes in Subsection 2.2.5 applies in the topological setting. 
3.1. The canonical transfer. In topology, the functor

$$
X \mapsto D X=\operatorname{Hom}(X, S),
$$

where $S$ is the identity of the stable homotopy category (the spherical spectrum), supplemented by its calculation for the spectra of certain spaces, is called the Spanier-Whitehead duality. A supplement to it is the Milnor-Spanier isomorphism $D \Sigma^{\infty} X_{\bullet} \simeq$ $\Sigma^{\infty} \operatorname{Th} N_{X}$ (see [22, Subsection 14.43]), where $X$ is a smooth variety, $X_{\bullet}$ is its one-point compactification, and $\Sigma^{\infty}$ Th $N_{X}=\Sigma^{-n} \Sigma^{\infty}$ Th $V$ for a bundle $V$ such that $T_{X}+V=1^{n}$ (see [22, Subsection 14.39]). In other words, $N_{X}=-T_{X}$ is a virtual bundle with $\operatorname{rk} N_{X}=-\operatorname{dim} X$.

A proper mapping $f: X \rightarrow Y$ extends to a mapping $f_{\bullet}: X_{\bullet} \rightarrow Y_{\bullet}$ of one-point compactifications, inducing an operator $D\left(\Sigma^{\infty} f_{\bullet}\right): D \Sigma^{\infty} Y_{\bullet} \rightarrow D \Sigma^{\infty} X_{\bullet}$. Passing to cohomology, we obtain an operator $\left(D \Sigma^{\infty} f_{\bullet}\right)^{*}: A\left(D \Sigma^{\infty} X_{\bullet}\right) \rightarrow A\left(D \Sigma^{\infty} Y_{\bullet}\right)$, which depends neither on the choice of an orientation nor on its existence. Using the above-mentioned duality for varieties, we obtain an operator $A\left(\Sigma^{\infty} \mathrm{Th} N_{X}\right) \rightarrow A\left(\Sigma^{\infty} \mathrm{Th} N_{Y}\right)$. Conditionally (the suspension spectrum of the corresponding Thom space is defined, rather than $N_{X}$ ), we can write this operator in the form

$$
\int_{f}: A\left(\operatorname{Th} N_{X}\right) \rightarrow A\left(\operatorname{Th} N_{Y}\right) .
$$

3.1.1. Construction of the canonical transfer. We prove that, for an orientable theory in the algebraic setting, there is a similar operator independent of the choice of an orientation. We assume that there is a functor $A\left(\operatorname{Th} N_{X}\right)$ and a natural isomorphism $A\left(\operatorname{Th} N_{X}\right) \otimes_{A(X)} A\left(\operatorname{Th} T_{X}\right) \rightarrow A(X)$. Such a functor is constructed by a realization of the stable normal bundle, i.e., by the set $\tilde{N}=\left(p: \tilde{X} \rightarrow X, i: p^{*} T_{X}+N \rightarrow 1^{n}\right)$, where $p$ is a composition of affine bundles [20] and $i$ is an isomorphism. It can be checked that, in essence, $A(\operatorname{Th} \tilde{N})=A(\operatorname{Th} N) \otimes C^{-n}$ does not depend on the realization, i.e., it is naturally isomorphic to the projective limit over all realizations, and we can define $A\left(\operatorname{Th} N_{X}\right)$ by this limit. Actually, the canonical transfer constructed below depends only on the identifications $A\left(\operatorname{Th} N_{X}\right) \otimes_{A(X)} A\left(\operatorname{Th} T_{X}\right) \rightarrow A(X)$ and $A\left(\operatorname{Th} N_{Y}\right) \otimes_{A(Y)}$ $A\left(\operatorname{Th} T_{Y}\right) \rightarrow A(Y)$, and the transfer is natural since these identifications are natural.

We define the Thom operator $\theta: A(X) \rightarrow A\left(\operatorname{Th} N_{X}\right) \otimes C^{-\operatorname{dim} X}$ so that $\theta \otimes_{A(X)} \theta^{T}=$ id (here $\theta^{T}$ is the Thom isomorphism for the tangent bundle $T$ ), and the canonical transfer $\int_{f}$ so that the following diagram is commutative:

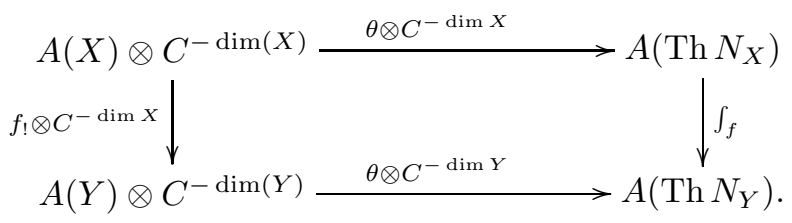

3.1.2. Theorem. The operator $\int_{f}$ does not depend on the orientation.

Proof. For the orientations given by parameters $u$ and $v$, we have the diagram

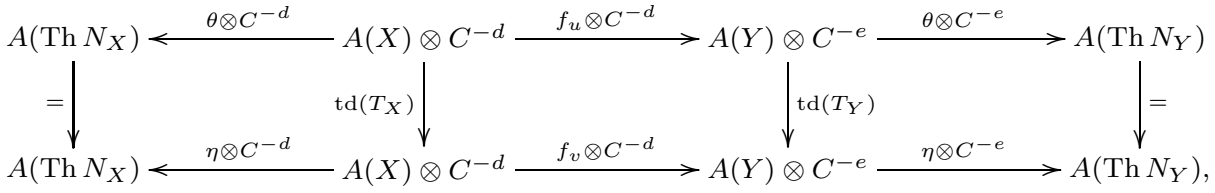

where $d=\operatorname{dim} X, e=\operatorname{dim} Y, \theta$ and $\eta$ are the Thom operators for fixed orientations of $A$ and $B$, and $\mathrm{td}=\mathrm{td}_{\mathrm{id}}$. The middle square is commutative by the Riemann-Roch 
theorem. The left (respectively, right) square is commutative because the termwise tensor multiplication by the square

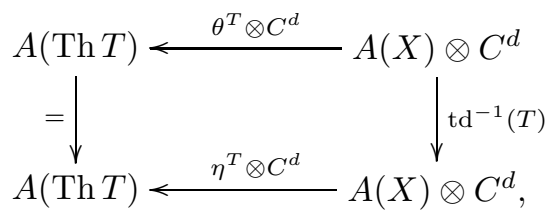

which is commutative by the Riemann-Roch theorem, leads to a commutative square (formed by identity arrows).

For a proper mapping $p: X \rightarrow Y$, the canonical transfer $\int_{p}$ can be regarded as integration along the "fibers" of the mapping $p$ (the "fibers" can be understood literally for a smooth mapping). To emphasize an analogy with integration of differential forms, we put $\Omega_{A}(X)=A\left(\operatorname{Th} N_{X}\right)$ and write integration along fibers (by abuse of notation, we replace $p$ with $X / Y)$ as

$$
\int_{X / Y}: \Omega_{A}(X) \rightarrow \Omega_{A}(Y) \text { and } \int_{X}: \Omega_{A}(X) \rightarrow R \text { for } Y=\text { pt. }
$$

The Thom isomorphism $\theta: A(X) \rightarrow \Omega_{A}(X) \otimes C^{-\operatorname{dim} X}$ induces the "volume form" $\theta_{X}=\theta(1) \in \Omega_{A}^{2 d, d}(X)$. In these terms, integration and transfer are related to each other as follows:

$$
p_{!}(\alpha) \theta_{Y}=\int_{X / Y} \alpha \theta_{X}
$$

3.2. The Riemann-Roch theorem and change of variables in integrals. Let $X$ be a smooth projective variety, and let $\theta=\theta_{X}$ and $\eta=\eta_{X}$. Then, by the RiemannRoch theorem for an operation $\phi: A \rightarrow B$ with orienting parameters $u$ and $\phi(u)$ and the operation id $: B \rightarrow B$ with parameters $\phi(u)$ and $v$, we have

$$
\phi \int_{X} \alpha \theta=\int_{X} \phi(\alpha) \phi(\theta)=\int_{X} \phi(\alpha) \frac{\phi(\theta)}{\eta} \eta .
$$

Furthermore,

$$
\phi(\theta) / \eta=j,
$$

where $j=\operatorname{td}_{\phi}(X)=j_{\phi}\left(N_{X}\right)$ (by the Riemann-Roch theorem for the mapping $X \rightarrow$ Th $T_{X}$ by zero). Moreover, if $N_{X}=L_{1}+\cdots+L_{d}$ (virtual expansion), then

$$
j=j\left(L_{1}\right) j\left(L_{2}\right) \cdots j\left(L_{d}\right) .
$$

To draw an analogy with the usual integration, we consider a topological homomorphism $\phi: A \rightarrow B$ of commutative topological algebras, a $d$-dimensional compact manifold $X$ with $A$-valued volume form $\theta$, and a nondegenerate $B$-valued volume form $\eta$. For a continuous function $\alpha: X \rightarrow A$, we have

$$
\phi \int_{X} \alpha \theta=\int_{X} \phi(\alpha) \phi(\theta)=\int_{X} \phi(\alpha) \frac{\phi \theta}{\eta} \eta
$$

Let $U$ be open in $X$. We fix diffeomorphisms $v: U \rightarrow V$ and $w: U \rightarrow W$, where $V$ is open in $\mathbb{R}^{d}$ with coordinates $v_{1}, \ldots, v_{d}$, and $W$ is open in $\mathbb{R}^{d}$ with coordinates $w_{1}, \ldots, w_{d}$. Assume that $\theta=v^{*}\left(d v_{1} \wedge \cdots \wedge d v_{d}\right)$ and $\eta=w^{*}\left(d w_{1} \wedge \cdots \wedge d w_{d}\right)$. Identifying the functions on $U$ and on $W$ with the help of $w$, we obtain

$$
\phi(\theta) / \eta=j,
$$


where $j$ is the Jacobian of the mapping $v \circ w^{-1}: W \rightarrow V$. Furthermore, if $V=$ $V_{1} \times \cdots \times V_{d}, W=W_{1} \times \cdots \times W_{d}$, and $v \circ w^{-1}$ is the direct product of the mappings $t_{i}: W_{i} \rightarrow V_{i}$, then

$$
j=\operatorname{jac}\left(t_{1}\right) \cdots \operatorname{jac}\left(t_{d}\right) .
$$

Formulas (24), (25), and (26) are similar to formulas (27), (28), and (29). Thus, the series $j_{\phi}$ can be regarded as the Jacobian of a change of parameters on $\mathbf{P}^{\infty}$, and the Riemann-Roch theorem can be thought of as a change-of-variables formula in integrals.

3.3. The Todd classes in topology. We show that the Todd classes used in the general topological Riemann-Roch theorem [4] can be calculated by formulas written in Subsection 2.2. The class $\tau(E)$ in [4] corresponds to the Todd class of the virtual bundle $(-E)$ used in [1] and in the present paper.

Let $\phi: A \rightarrow B$ be a stable multiplicative operation between cohomology theories of appropriate topological spaces (see $[22,7]$ ). We assume (this is convenient because appropriate references are available) that $A$ and $B$ are represented by ring spectra and that $\phi$ is a ring operation between them (see $[22,9]$ ).

Suppose that $A$ and $B$ are oriented and that $x$ and $y$ are the orienting parameters (see $[23$, p. 395], where a parameter is called an orientation of the theory in question). In this case, every complex bundle $E$ is equipped with an orientation (a Thom isomorphism) $\omega_{x}$ : $A^{0}(X) \rightarrow A^{n}(\mathrm{Th} E)$, where $n=\operatorname{rk} E$; see [23, Chapter VII, Subsection 2.8]. For example, $\omega_{x}(1)=u_{x}(t)$ for the universal line bundle $L=\mathcal{O}(1) / \mathbb{C} \mathbf{P}^{\infty}$, where $t \in M U^{2}\left(\mathbb{C} \mathbf{P}^{\infty}\right)$ is given in [23, Chapter VII, after Subsection 2.4] and $u_{x}: M U \rightarrow A$ is the ring morphism determined by $x$ [23, Chapter VII, Subsection 2.7]. In their turn, the orientations $\omega_{x}$ and $\omega_{y}$ of the bundle $E$ determine (see [4]) the class $\tau(E)$ as the image of the identity under the composition

$$
A^{0}(X) \stackrel{\omega_{x}}{\longrightarrow} A^{2 \mathrm{rk} E}(\mathrm{Th} E) \stackrel{\phi}{\rightarrow} B^{2 \mathrm{rk} E}(\mathrm{Th} E) \stackrel{\omega_{y}^{-1}}{\longrightarrow} B^{0}(X)
$$

where Th $E$ is the Thom space. Thus, for a complex manifold $X$ we have the class $\tau(X)=\tau(N)$, where $N$ is the stable normal bundle of $X$, i.e., $N=E / T$, where $E$ is the trivial bundle and $T$ is the tangent bundle of $X$ embedded in $E$. Since $\phi$ is stable, the Todd class $\tau(N)$ depends neither on the choice of $E$ nor on an embedding $T \subset E$.

Let $\phi: K \rightarrow H^{*}(X, \mathbb{Q})[[d]]$ be the Chern character, where $\operatorname{deg} d=-2$ (we do not discuss the extendibility of ch from $K_{0}$ to the higher $K$-theory; representability follows from [22, Subsection 9.24]). In this case, we have a simple formula (see [1, Chapter III, $\S 10])$ calculating $\tau(E)$ by the Chern classes. Specifically, $\tau(E)=j\left(\lambda_{1} d\right) \cdots j\left(\lambda_{n} d\right)$, where

$$
j(z)=\frac{e^{z}-1}{z}
$$

and formally $c_{0}(E)+\cdots+c_{n}(E) d^{n}=\left(1+\lambda_{1} d\right) \cdots\left(1+\lambda_{n} d\right)$ with $n=\operatorname{rk} E$.

Theorem 3.3.1 shows that we also have a simple formula for a general operation $\phi$. Since the Todd classes are multiplicative (this follows from the multiplicativity of the Thom classes [23, p. 398] and the splitting principle [23, Chapter VII, Subsection 2.2]), it suffices to present a formula for the universal line bundle $L=\mathcal{O}(1) / \mathbb{C} \mathbf{P}^{\infty}$.

3.3.1. Theorem. We have $\tau(L)=j(y)$, where the series $j$ is defined by the same formula as in Subsection 2.2.1, i.e.,

$$
j(y)=\phi(x) / y \text {. }
$$

Proof. We need the following auxiliary statement. Consider the diagram

$$
A^{0}(H) \stackrel{\omega_{x}}{\longrightarrow} A^{2}(\mathrm{Th} L) \stackrel{i_{A}}{\longrightarrow} A^{2}\left(\mathbb{C} \mathbf{P}^{\infty}\right),
$$


where $H$ is a hyperplane in $\mathbb{C P}^{\infty}$ and $i_{A}=s \circ e^{-1}$ is the composition of the inverse isomorphism for the excision $e$ and the extension of supports $s$ in the diagram

$$
A^{2}(\operatorname{Th} L)=A^{2}\left(\mathbb{C} \mathbf{P}^{\infty}-P, \mathbb{C} \mathbf{P}^{\infty}-H-P\right) \stackrel{e}{\leftarrow} A^{2}\left(\mathbb{C} \mathbf{P}^{\infty}, \mathbb{C} \mathbf{P}^{\infty}-H\right) \stackrel{s}{\rightarrow} A^{2}\left(\mathbb{C} \mathbf{P}^{\infty}\right) .
$$

Here $L$ is regarded as a bundle on $H, P \in \mathbb{C} \mathbf{P}^{\infty}$ is a point outside of $H$, and equality comes from the varieties $L \simeq \mathbb{C} \mathbf{P}^{\infty}-P$. We claim that

$$
i_{A} \circ \omega_{x}(1)=x \text {. }
$$

Comparing (32) with a similar sequence for $M U$ with the help of $u_{x}$, and using the fact that $L / \mathbb{C} \mathbf{P}^{\infty}$ can be taken as the universal bundle on $B U_{1}$, we reduce the problem to the relation $i_{M U} \circ \omega_{t}(1)=t$, which, in essence, coincides with the definition of $t$ (see $\left[23\right.$, p. 396, after (2.4)]) and the construction of the Thom operator $\omega_{t}$ (see $[23$, p. 397, Subsection 2.8]).

Now, we derive (31) from (33). By definition, $\tau(L)=\omega_{y}^{-1} \circ \phi \circ \omega_{x}(1)$, where $\omega_{x}$ and $\omega_{y}$ are the Thom isomorphisms for the bundle $L$. Next, $\omega_{y}^{-1} \circ \phi \circ \omega_{x}(1)=\omega_{y}^{-1} \phi\left(i_{A}^{-1} x\right)=$ $\omega_{y}^{-1}\left(i_{B}^{-1} \phi x\right)=\omega_{y}^{-1}\left(i_{B}^{-1}(j(y) y)\right)=\omega_{y}^{-1}\left(j(y) \omega_{y}(1)\right)=j(y)$.

\section{REFERENCES}

[1] F. Hirzebruch, Topological methods in algebraic geometry, Grundlehren Math. Wiss., Bd. 131, Springer-Verlag New York, Inc., New York, 1966. MR0202713 (34:2573)

[2] A. Borel and J.-P. Serre, Le théorème de Riemann-Roch, Bull. Soc. Math. France 86 (1958), 97-136. MR0116022 (22:6817)

[3] M. F. Atiyah and F. Hirzebruch, Riemann-Roch theorems for differentiable manifolds, Bull. Amer. Math. Soc. 65 (1959), 276-281. MR0110106 (22:989)

[4] E. Dyer, Relations between cohomology theories, Colloquium on Algebraic Topology (August 1-10, 1962), Various Publ. Series, No. 1, Mat. Inst. Aarhus Univ., Aarhus, 1962, pp. 89-93.

[5] A. Suslin and V. Voevodsky, Bloch-Kato conjecture and motivic cohomology with finite coefficients, The Arithmetic and Geometry of Algebraic Cycles (Banff, AB, 1998), NATO Sci. Ser. C Math. Phys. Sci., vol. 548, Kluwer Acad. Publ., Dordrecht, 2000, pp. 117-189. MR1744945 (2001g:14031)

[6] F. Morel and V. Voevodsky, Homotopy category of schemes over a base, Preprint, 1997.

[7] V. Voevodsky, A $\mathbf{A}^{1}$-homotopy theory, Doc. Math. 1998, Extra Vol. 1, 579-604. MR1648048 (99j:14018)

[8] I. Panin and A. Smirnov, Push-forwards in oriented cohomology theories of algebraic varieties, K-Theory Preprint Archives no. 459, 2000.

[9] I. Panin, Oriented cohomology theories of algebraic varieties, K-Theory 30 (2003), 265-314. MR2064242 (2005f:14043)

[10] A. L. Smirnov, Orientations and transfers in the cohomology of algebraic varieties, Algebra i Analiz 18 (2006), no. 2, 167-224; English transl., St. Petersburg Math. J. 18 (2007), no. 2, 305-346. MR2244939 (2007f:14018)

[11] I. Panin, Riemann-Roch theorems for oriented cohomology, Axiomatic, Enriched and Motivic Homotopy Theory, NATO Sci. Ser. II Math. Phys. Chem., vol. 131, Kluwer Acad. Publ., Dordrecht, 2004, pp. 261-333. MR2061857 (2005g:14025)

[12] H. Miller, Universal Bernoulli numbers and the $S^{1}$-transfer, Current Trends in Algebraic Topology. Part 2 (London Ont., 1981), CMS Conf. Proc., vol. 2, Amer. Math. Soc., Providence, RI, 1982, pp. 437-449. MR0686158 (85b:55029)

[13] M. Hovey, Model categories, Math. Surveys Monogr., vol. 63, Amer. Math. Soc., Providence, RI, 1999. MR1650134 (99h:55031)

[14] V. Voevodsky, Reduced power operations in motivic cohomology, Publ. Math. Inst. Hautes Études Sci. No. 98 (2003), 1-57. MR2031198 (2005b:14038a)

[15] H. Gillet, Riemann-Roch theorems for higher algebraic K-theory, Adv. in Math. 40 (1981), 203-289. MR0624666 (83m:14013)

[16] Ch. Soulé, Opérations en K-théorie algébrique, Canad. J. Math. 37 (1985), 488-550. MR0787114 (87b:18013)

[17] V. M. Bukhshtaber, New methods in cobordism theory, R. Stong. Notes on Cobordism Theory, "Mir", Moscow, 1973, Appendix to the Russian ed., pp. 336-365. (Russian) MR0346789 (49:11513)

[18] M. F. Atiyah and F. Hirzebruch, Cohomologie-Operationen und charakteristische Klassen, Math. Z. 77 (1961), 149-187. MR0156361 (27:6285) 
[19] W. Fulton, Intersection theory, 2nd ed., Ergeb. Math. Grenzgeb. (3), Bd. 2, Springer-Verlag, Berlin, 1998. MR1644323 (99d:14003)

[20] A. L. Smirnov, Homotopy properties of algebraic vector bundles, Zap. Nauchn. Sem. S.-Peterburg. Otdel. Mat. Inst. Steklov. (POMI) 319 (2004), 261-263; English transl., J. Math. Sci. (N.Y.) 134 (2006), no. 6, 2580-2581. MR2117860 (2005i:14025)

[21] R. Hartshorne, Algebraic geometry, Grad. Texts in Math., No. 52, Springer-Verlag, New YorkHeidelberg, 1977. MR0463157 (57:3116)

[22] R. M. Switzer, Algebraic topology-homotopy and homology, Grundlehren Math. Wiss., Bd. 212, Springer-Verlag, New York-Heidelberg, 1975. MR0385836 (52:6695)

[23] Yu. B. Rudyak, On Thom spectra, orientability, and cobordism, Springer-Verlag, Berlin, 1998. MR1627486 (99f:55001)

St. Petersburg Branch, Steklov Mathematical Institute, Russian Academy of Sciences, Fontanka 27, St. Petersburg 191023, Russia

E-mail address: smirnov@pdmi.ras.ru

Received 29/MAY/2006

Translated by B. M. BEKKER 